Mots. Les langages du politique

$96 \mid 2011$

Les discours politiques. Approches interactionnistes et multimodales

\title{
La dénomination comme trace du passé et enjeu. L'exemple des universités parisiennes
}

\section{Christine Barats}

\section{OpenEdition}

\section{Journals}

Édition électronique

URL : https://journals.openedition.org/mots/20250

DOI : $10.4000 /$ mots. 20250

ISSN : 1960-6001

Éditeur

ENS Éditions

Édition imprimée

Date de publication : 5 septembre 2011

Pagination : 65-80

ISBN : 978-2-84788-316-9

ISSN : 0243-6450

Référence électronique

Christine Barats, «La dénomination comme trace du passé et enjeu. L'exemple des universités parisiennes », Mots. Les langages du politique [En ligne], 96 | 2011, mis en ligne le 05 septembre 2013, consulté le 22 avril 2022. URL : http://journals.openedition.org/mots/20250 ; DOI : https://doi.org/ $10.4000 /$ mots. 20250 


\section{Christine Barats}

\section{La dénomination comme trace du passé et enjeu. L'exemple des universités parisiennes}

Depuis le début des années 2000, la dénomination des universités a été inscrite à l'agenda des préoccupations de l'enseignement supérieur'1. De nombreuses universités françaises ont changé de nom, soulignant par cet acte de langage - où dire, c'est faire exister² - l'acuité de la dénomination dans un contexte de réformes et de mutations du supérieur (logique de regroupement entre anciennes facultés puis entre différentes universités : PRES - pôles de recherche et d'enseignement supérieur-, Plan Campus). Les universités dites parisiennes, atypiques dans l'espace académique national par leur concentration notamment, illustrent cependant les enjeux actuels du supérieur quant à l'importance du nom en tant que vecteur de l'image, voire de la marque, des établissements universitaires. Nous nous proposons donc d'examiner d'un point de vue diachronique, dans la moyenne durée, les caractéristiques morphosyntaxiques et sémantiques des changements de nom des treize universités dites parisiennes3. Pour saisir les traces d'emploi de ces dénominations depuis 1970, nous avons privilégié différents corpus relevant des espaces juridiques, administratifs et de communication : textes réglementaires, documents internes à l'établissement, articles de presse, communiqués de presse, sites web. Ces corpus nous permettront d'examiner les logiques juridico-administratives dans un premier temps, puis essentiellement communicationnelles dans la construction des dénominations.

1. Par commodité, nous utiliserons «supérieur » pour « enseignement supérieur».

2. Perspective pragmatique telle que l'envisageait John Langshaw Austin dans Quand dire c'est faire (1970).

3. Nous avons retenu, par souci d'homogénéité, les treize universités nées après les réformes de 1968 et qui utilisent ou utilisaient une référence à «Paris» dans leur nom. Nous avons donc exclu les quatre universités créées en 1991 (Cergy-Pontoise, Évry-Val-d’Essonne, Paris-Est Marne-laVallée et Versailles Saint-Quentin-en-Yvelines) afin de répondre à la massification de l'enseignement supérieur et à l'accueil de milliers de nouveaux étudiants. Elles sont cependant situées, comme d'autres universités qualifiées de «parisiennes» (Paris VIII, IX, X...), à la périphérie de Paris. Nous avons privilégié des logiques historiques et administratives plutôt que des logiques strictement géographiques (Paris vs banlieue).

Céditec, UPEC et Université Paris Descartes

christine.barats@parisdescartes.fr

Mots. Les langages du politique $\mathrm{n}^{\circ} 96$ juillet $2011 \bullet 65$ 
Si les noms d'établissements académiques occupent une place particulière dans l'espace discursif des noms propres4, compte tenu du poids des logiques juridico-administratives et communicationnelles dans la construction du nom, ils sont, aujourd'hui, soumis à des consignes de la part des équipes présidentielles afin de stabiliser leur usage et de bénéficier ainsi d'une meilleure comptabilisation des signatures et d'un meilleur référencement. La notoriété dépend en effet de l'importance accordée à la place des universités françaises dans les classements internationaux 5 et de la généralisation des outils technologiques de référencement qui induisent une stabilisation de la dénomination afin qu'elle soit cumulée (moteurs de recherche et outils bibliométriques).

Après avoir examiné les caractéristiques morphosyntaxiques et «l'épaisseur sémantique» (Barthes, 1967) des treize dénominations académiques étudiées, nous nous intéresserons aux logiques de regroupement entre établissements et à la diversité des dénominations au regard de la question de l'affiliation. Nous accorderons une attention toute particulière aux récents changements de noms et aux usages du nom Sorbonne, signes de la prise en compte d'une logique de marque.

\section{Approche généalogique. Pluralité des dénominations et ancrage topologique dans le champ juridico-administratif}

Il y a en France, en 2010, 83 universités et établissements assimilés qui sont liés à la loi Faure du 12 novembre 1968. Ce nombre peut varier, selon les sources, de $83^{6}$ à $85^{7}$, ce qui souligne déjà l'importance de la nomenclature et des dénominations dans l'histoire des organismes d'enseignement et de recherche dans le supérieur français ${ }^{8}$. Antérieurement, les établissements étaient structurés par champ disciplinaire, selon une division facultaire (Musselin, 2001, p. 103 et suiv.). La loi Faure du 12 novembre 1968 a supprimé les anciennes facultés au profit d'une logique universitaire d'établissements souvent pluridisciplinaires, donnant naissance, au-dessus des entités disciplinaires, à des

4. Voir les numéros 63 (Honoré, Paveau, Périès éd., 2000), 86 (Boyer, Paveau éd., 2008) et 87 (Bacot, Douzou, Honoré éd., 2008) de la revue Mots. Les langages du politique et le numéro 11 (Paveau, Reboul-Touré, Lecolle éd., 2009) des Carnets du Cédiscor.

5. La notoriété des universités dépend, en particulier, du classement dit «de Shanghai », qui s'est imposé dans l'espace médiatique nonobstant les critiques formulées quant à son élaboration même. Pour rappel, «comparabilité » des universités et choix des principaux critères : obtention de prix Nobel ou de médailles Fields, citation des chercheurs dans la revue Nature, Science, etc. Ce classement mondial des universités a été élaboré parl'université Jiao Tong de Shanghai, qui publie depuis 2003, au mois d'août, un classement des «meilleures universités ». Voir Gingrat, 2008.

6. Source : site du ministère de l'Enseignement supérieur.

7. Source : presse nationale, rapports officiels et sites, par exemple site de "Sauvons la recherche».

8. L’université Paris IX est un « grand établissement » depuis février 2004. Nous emploierons dans le corps de cet article « université » pour les treize établissements étudiés. 
établissements publics à caractère scientifique et culturel (EPSC) désignés génériquement par le nom d'universités9. Néanmoins, l'administration centrale a continué à prendre ses décisions «en fonction de la situation de la discipline concernée par rapport à l'ensemble du territoire, plutôt qu'en tenant compte de la situation particulière de chaque établissement, et les universités restaient absentes des discours, des représentations et des pratiques des agents du ministère » (ibid., p. 103). En mars 1989, une circulaire ${ }^{10}$ déterminera la politique dite contractuelle. Elle met en place l'autonomisation des établissements du supérieur qui négocient, tous les quatre ans, avec l'autorité de tutelle. La base de la négociation est le projet d'établissement qui donne lieu à la signature, entre l'établissement et l'autorité de tutelle, d'un contrat quadriennal. Si, schématiquement, la loi Faure rompt avec une logique facultaire au profit d'universités regroupant des UFR (Unités de formation et de recherche, souvent les ex-«facultés») et des instituts, la politique contractuelle de 1989 renforcera cette logique en modifiant les relations entre l'État et les établissements. Dès 1970 et jusqu'en 1971, plusieurs décrets vont réorganiser l'ancienne Université de Paris et créer de nouvelles entités dont le nom administratif renvoie à la numérotation des universités parisiennes après 1971, de un à treize. Soulignons que la réorganisation de l'espace académique parisien, à la fin des années 1960, s'inscrivait dans un découpage de domaines de compétence et de références théoriques non exempt de luttes politiques, idéologiques et syndicales caractéristiques de cette période. Un premier corpus, constitué des arrêtés d'approbation des statuts ${ }^{11}$, atteste d'une dénomination articulant «Université de Paris » + chiffre romain (voir tableau en annexe). Certaines dénominations sont accompagnées d'une discipline (Paris $\left.\right|^{12}$ et II), d'un nom d'auteur (Paris V) ou d'un ancrage topologique (Sorbonne, Panthéon...). En avril 2010, la construction toponymique domine, même si deux universités, sur les treize étudiées, ne font plus référence ni à la ville de Paris, ni au numéro sur leurs sites web : «Université Panthéon-Assas » et «Université Pierre et Marie Curie ». L' "Université Paris Descartes » et l' "Université Paris Diderot » ont conservé dans leur dénomination la référence à «Paris».

D'un point de vue diachronique, l'ancrage topologique du nom caractérise les dénominations des six universités dites parisiennes (pourtant situées

9. Ils deviendront «établissement public à caractère scientifique, culturel et professionnel» (EPSCP) en 1984. Nous pouvons noter le cas particulier des facultés de médecine qui ont conservé leur «identité » hospitalière et leurs pouvoirs et hiérarchies administratives.

10. Circulaire no 89-079 du 24 mars 1989, Bulletin officiel $n^{\circ} 13$ du 30 mars 1989, p. 761-765.

11. Les sources juridico-administratives attestant de l'emploi des dénominations sont nombreuses. Une recension exhaustive s'avère difficile. Nous avons retenu tous les arrêtés d'approbation des statuts pour identifier les dénominations en cours au moment de la création de ces établissements. Nous avons indiqué dans le tableau en annexe la dénomination figurant dans le titre, ainsi que celles figurant dans les articles de l'arrêté, lorsqu'elles se distinguent de celle du titre.

12. Par convention, lorsqu'une université étudiée est citée dans l'article à titre d'exemple, nous employons « Paris» + numéro en chiffre romain. 
à l'extérieur de Paris) créées après 1968 (corpus : arrêtés d'approbation des statuts). Elles illustrent la logique dominante dans la construction des dénominations académiques, à savoir une construction morphosyntaxique articulant une logique toponymique (ancrage territorial) 13 à un numéro ${ }^{14}$ :

- «Université de Paris-Vincennes, Paris-VIII », en référence au bois de Vincennes ${ }^{15}$, son lieu de création, parfois encore appelée «ex-Vincennes » par allusion à la période symbolique d'effervescence intellectuelle liée à sa création ${ }^{16}$;

- «Université de Paris-Dauphine, Paris-IX» en référence à la Porte Dauphine;

- «Université de Paris-Nanterre, Paris-X», ville d'implantation de l'université;

- «Université de Paris-Sud, Paris-XI», liée à la faculté des sciences d'Orsay, ville d'implantation, aussi désignée par «Université d'Orsay»;

- "Université de Paris Val-de-Marne, Paris-XII », appelée aussi "Université de Créteil », ville nouvelle d'implantation de l'université, ou encore "Université Paris 12 Val-de-Marne»;

- «Université Paris-Nord, Paris XIII», en référence à sa situation géographique ${ }^{17}$.

Si les dénominations académiques se sont principalement construites, dans le champ juridico-administratif, selon une logique toponymique, les noms en usage pour désigner un même établissement s'avèrent pluriels et constituent des traces de l'histoire de ces établissements ainsi que des relations que les locuteurs ont nouées ou nouent avec ces entités.

L'usage du chiffre romain dans la dénomination était dominant jusqu'au milieu des années 1980. Il a été majoritairement remplacé par un chiffre arabe sur les documents de communication (affiches, courriers...) ${ }^{18}$. Six universités (Paris II, V, VI, IX, X et XII) ne font plus référence à un chiffre sur leurs supports de communication, signe d'une rupture par rapport aux logiques antérieures (corpus : sites web). Le passage, dans les années 1990, du chiffre romain au chiffre arabe coïncide avec la prise en compte de stratégies de communication et la multiplication de discours d'accompagnement marqués par la rhéto-

13. Les quatre universités créées en 1991 ont privilégié cette seule logique dans le choix de leur nom : Université de Cergy-Pontoise, Université d’Évry-Val-d’Essonne, Université Paris-Est Marne-la-Vallée et Université de Versailles Saint-Quentin-en-Yvelines.

14. Cette structure s’observe également dans le cas des dénominations des universités de province : Aix-Marseille I, Aix-Marseille II, etc., dénominations également en cours de changement, indices de mutations qui touchent l'ensemble du supérieur.

15. Le «Centre universitaire expérimental de Vincennes » a été construit dans le bois de Vincennes et a été rasé en 1979.

16. L'expression «ex-Vincennes» rappelle le lieu qui a accueilli Michel Foucault en 1968, Gilles Deleuze et Jean-François Lyotard (de 1970 à 1987) ainsi que François Châtelet, pour ne citer que ces auteurs qui ont contribué à la notoriété de Paris VIII.

17. Ce qui permet d'éviter la référence à la Seine Saint-Denis.

18. Le difficile accès aux sources (problème des archives), en particulier aux sources internes aux établissements nécessiterait, pour compléter cette étude, un travail monographique et ethnographique afin de dater précisément les périodes de changement (comptes rendus des conseils d'administration, de réunions, etc.). 
rique de la "modernité» attendue dans le supérieur. Nous avons examiné les adresses des sites web (URL) des établissements étudiés, ainsi que la place du nom dans la composition graphique de la page d'accueil (avril 2010 ; voir Barats, à paraître). La syntaxe des URL indique une construction comparable à celle qui caractérise la dénomination dans le champ juridico-administratif, à savoir : www.univ-paris + chiffre arabe.fr, et ce, indépendamment des changements de nom ${ }^{19}$. Il n'y a cependant pas de norme commune aux treize universités, à l'inverse d'autres pays qui indiquent dans leur URL, par les lettres « edu » ou «ac», l'appartenance au champ éducatif ou académique du site (ÉtatsUnis, Grande-Bretagne, Belgique) ${ }^{20}$. C'est la place stratégique du nom dans le site (bandeau supérieur + logo) qui inscrit le site dans le champ académique.

Seule l'université Paris IV a gardé le chiffre romain dans ses documents de communication et sur son site ${ }^{21}$ : elle a changé de nom en 2007 au profit d' « Université Paris Sorbonne - Paris IV». "Signe volumineux» pour Roland Barthes, le nom propre témoigne d'une «épaisseur sémantique » dont le feuilleté sémique produit une «hypersémanticité » (Barthes, 1967, p.122). Le choix des noms propres fonde ainsi le récit chez Proust (ibid., p. 128). Il forme un système : «tenir le système des noms, c'était pour Proust [...] tenir les significations essentielles du livre». Cette hypermotivation du signe ne s'observe pas uniquement dans le champ de la littérature, où le choix du nom propre fait l'objet de toutes les attentions. C'est également le cas lorsqu'il s'agit de dénommer une institution et de construire par ce biais son image : les traces de cette hypermotivation circulent alors non plus dans l'imaginaire d'un auteur et de ses lecteurs par le biais des désignations romanesques, mais dans l'espace social au moyen de supports de communication comme le papier à en-tête, les affiches, les sites web et les logotypes qui mettent en scène le nom et son «épaisseur sémantique ${ }^{22}$. Dans le champ académique, le choix du chiffre romain contribue ainsi au «feuilleté sémique » qu'évoque le nom : il est en effet porteur de références symboliques (le latin) et historiques (histoire médiévale de l'Universitas et du «quartier latin»).

Trois établissements sur les treize étudiés ont associé le nom administratif historique (constitué par « Université Paris + chiffre romain puis chiffre arabe ») au patronyme de personnalités françaises internationalement reconnues (Descartes, Diderot, Pierre et Marie Curie). Cette référence à des personnalités

19. À l'exception de Paris IV (www.paris-sorbonne.fr), Paris VI (www.upmc.fr), Paris VII (www.univparis-diderot.fr), Paris IX (www.dauphine.fr) et Paris XI (www.u-psud.fr). Nous n'aborderons pas la question de l'adresse électronique des enseignants-chercheurs; notons également une absence de norme dans ce domaine et la coexistence, à travers le temps, de plusieurs adresses pour un même enseignant et une même université.

20. Les sites des différents ministères français sont ainsi identifiables par la présence de «.gouv.fr » dans leur URL.

21. C'était également le cas sur les sites de Paris III et Paris X jusqu'à la fin 2009.

22. Pour une étude sémiologique et communicationnelle des sites web, voir Barats, à paraître. 
incarnant le "génie» intellectuel français est censée contribuer à construire une légitimité académique comparable au rôle joué par le chiffre romain ou le nom Sorbonne.

Cependant, les contraintes médiatiques ou les effets des présentations en liste ne rendent pas compte de cette «épaisseur sémantique » en optant pour une dénomination que nous pourrions qualifier d'administrative, car liée aux relations entre les établissements et l'autorité de tutelle et caractérisée par «lieu + chiffre arabe». La presse nationale et les dépêches d’agence illustrent ce choix de dénomination (corpus : Le Monde, Le Figaro, Libération, juillet 2008). Citons pour exemple une dépêche de l'AFP de juillet 2008, reprise dans la presse quotidienne nationale et qui utilisait «Paris + chiffre arabe» pour désigner les universités qui «passaient à l'autonomie» :

PARIS (AFP, 23 juillet 2008) - La ministre de l'Enseignement supérieur, Valérie Pécresse, a arrêté mercredi la liste des 20 premières universités qui accéderont à l'autonomie au $1^{\mathrm{er}}$ janvier 2009. [...] 20 universités pilotes, sur les 85 existantes, vont devenir autonomes et pourront ainsi gérer leur budget elles-mêmes. Les 20 universités concernées sont Aix-Marseille 2, Cergy-Pontoise, Clermont-Ferrand 1, Corte, Limoges, Lyon 1, Marne-la-Vallée, Montpellier 1, Mulhouse, Nancy 1, Paris 5, Paris 6, Paris 7, La Rochelle, Saint-Étienne, Strasbourg 1, Strasbourg 2, Strasbourg 3, Toulouse 1 et l'université technologique de Troyes.

Si le choix de cette structure de dénomination est lié à la présentation en liste, rappelons que les sites et les services de communication de ces universités utilisaient à la même date d'autres noms pour s'autodésigner. Il y a donc coexistence de différents noms pour une même université à un temps «t»: le nom diffusé et promu par l'université, le nom administratif ou le nom historique, les différents noms d'usage 23 qui peuvent également varier en fonction du locuteur (sociographie d'acteurs et type d'interaction sociale), du lieu de discours (autorité de tutelle, presse, université) ou de la situation de communication (professionnelle, non professionnelle, etc.), ou bien encore en fonction de la période au cours de laquelle le locuteur a été en lien avec l'université (le nom comme trace d'une période de formation, par exemple).

La pluralité de noms des universités est donc le fruit de cette sédimentation historique, des différentes politiques publiques et de la relation que le locuteur construit discursivement avec l'entité désignée, d'où la coexistence de plusieurs noms pour un même établissement, et ce, depuis leur création. Les différents noms en usage sont les traces de cette longue construction (référence à un lieu, à un auteur, à un chiffre, à une composante).

L'université Paris XII, qui avait adopté comme signature : «Université Paris 12 - Val de Marne» (supprimant le chiffre romain), a changé de nom en octobre 2009

23. Il est difficile de faire une recension exhaustive des différents noms ou expressions qui ont été en usage ou qui le sont encore. Nous avons privilégié certains espaces. 
pour «Université Paris-Est Créteil Val de Marne » (supprimant toute référence au chiffre). Elle est cependant aussi désignée par «Université de Créteil » ou bien par les noms de ses composantes, comme «l'IUT Sénart-Fontainebleau » ou «l'IUT de Créteil-Vitry »24. L'adhésion, en juillet 2007, au PRES (Pôle de recherche et d'enseignement supérieur) 25 dénommé «Université Paris-Est » est présentée, en octobre 2009, comme l'une des raisons expliquant cette «évolution » de nom. Il est intéressant de noter que le courrier de la présidente de Paris XII26, précédant le vote du conseil d'administration, réfute l'idée de «changement » : "Le nom ne devait pas “changer”, mais évoluer. II ne devait pas marquer un changement dans notre positionnement, nos valeurs collectives ou dans notre projet commun, mais une évolution positive nous permettant de mieux nous affirmer sur nos territoires et dans cet ensemble que nous avons rejoint. »

Les logiques de regroupement liées aux PRES renforcent ainsi l'importance de la dénomination tout en contribuant à une multiplication des signatures.

Entre 2007 et 2009, sept établissements, sur les treize étudiés, ont changé de nom et deux établissements ont examiné cette éventualité (Paris I et III). Le choix des nouvelles dénominations (corpus : sites web, avril 2010) indique une prise en compte de logiques de communication, voire de marque. L'université Paris IV a ainsi adopté en 2007 comme signature : «Université Paris Sorbonne - Paris IV», dans une logique de marque, comme nous l'étudierons ci-après. L'université Paris II a opté pour le lieu : «Panthéon-Assas». Le nom «Université Panthéon-Assas» est associé sur le site web à un slogan : "Première université de Droit de France »27, référence explicite à la notion de palmarès et au discours dit de l'excellence. L'université Paris V a privilégié le choix d'un anthroponyme au rayonnement international et lié au champ académique : «Université Paris Descartes », comme les universités Paris VI et VII, respectivement «Université Pierre et Marie Curie » et «Université Diderot». L'université Paris X a opté pour : «Université Paris Ouest Nanterre La Défense » afin de souligner sa proximité géographique avec le site de La Défense, un des principaux quartiers d'affaires de la région parisienne. Le changement de nom s'est accompagné d'un changement d'image : «Plus moderne, plus ambitieuse, plus dynamique, cette nouvelle image correspond aux nouveaux enjeux de l'université : un nom

24. Dans ce cas, le nom des composantes élude le nom de l'ensemble; il ne s'agit cependant pas du même référent.

25. Loi de programme du 18 avril 2006 sur la recherche; 9 PRES créés par décret en mars 2007.

26. Courrier de Simone Bonnafous, à l'attention des «collègues, étudiants et partenaires de l'université », daté du 16 octobre 2009 et accompagnant le rapport de l'agence de communication Campus Communication qui a été chargée de la réflexion sur l'évolution du nom. Le communiqué de presse de janvier 2010 souligne : «L'Université Paris 12 devient l'UPEC. Autonome depuis le 1 er janvier 2010, l'Université Paris 12 - Val de Marne est devenue, dans le même temps, l'Université Paris-Est Créteil Val de Marne, l'UPEC. Cette évolution de nom répond aux choix stratégiques inscrits dans le projet d'établissement 2009-2012.»

27. Notons que le droit n'est pas l'unique cursus de cette université; le slogan tend à associer au nom une identité disciplinaire. 
connu internationalement, une place de leader dans les sciences humaines et un enracinement territorial en développement. $»^{28}$

Dans ce registre qui se veut communicationnel, il est intéressant de souligner que la prise en compte de logiques de communication s'est accompagnée d'une diffusion de sigles. Cinq universités (Paris V, VI, X, XII et XIII) ont ainsi adopté les sigles suivants : URD (pour "Université René Descartes», attesté dans de nombreux documents internes entre 2000 et 2007), UPMC (pour Université Pierre et Marie Curie, sigle qui figure depuis 2008 sur le site web et qui est à la base de son URL), UPX, UPEC et UP13 (sites web respectifs).

Le nombre de sites (référence aux lieux) ou de composantes (référence aux anciennes facultés ou UFR), l'histoire des établissements et la structuration du supérieur français sont autant d'éléments qui expliquent la coprésence de plusieurs noms pour désigner une même entité. Or cette situation, amplifiée par les changements de nom, constitue un handicap dans le contexte de discours de l'excellence basés sur le rang occupé dans les classements internationaux.

\section{Améliorer son rang. L'affiliation comme enjeu et les logiques de regroupement}

La médiatisation du classement dit de Shanghai, l'un des plus connus, a renforcé les préoccupations quant à la notoriété des établissements, à leur «compétitivité » en termes de rang obtenu, ce qui conduit à des regroupements. L'autorité de tutelle mène une politique d'incitation à travers différents dispositifs : PRES, fondations ou Plan Campus 29 (janvier 2008). Ce derniervise à rassembler plusieurs universités ou plusieurs PRES dans le but « de développer des pôles d'excellence de formation et de recherche «30. Dix pôles universitaires d'excellence de niveau international sont escomptés. En juillet 2008, la ministre de l'Enseignement supérieur, Valérie Pécresse, a rendu publique la liste des universités et grandes écoles qui étaient retenues pour constituer ces Campus et bénéficier d'importants financements (6 milliards d'euros annoncés en 2008). Dans un contexte de concurrence entre établissements et/ou pôles, les noms administratifs adoptés par les différents regroupements contribuent à l'institutionnalisation et à la mise en mots des ensembles ainsi constitués. Dans cet ordre de discours ${ }^{31}$, les noms des regroupements sont censés incarner la nouvelle entité, la faire exister en la nommant. Il n'est pas rare que ces associations entre établissements évoluent dans le temps. Ainsi, depuis 2006, les

28. Communiqué de presse de septembre 2009.

29. Àne pas confondre avec les appels à projets «Campus numériques», de 2000, 2001, 2002 et 2003.

30. Il s'agit d'un des quatre critères de sélection des projets. Source : site du ministère de l'Enseignement supérieur et de la Recherche.

31. "Le discours n'est pas simplement ce qui traduit les luttes ou les systèmes de domination, mais ce pour quoi, ce par quoi on lutte, le pouvoir dont on cherche à s'emparer. » (Foucault, 1971, p.12). 
alliances et leurs noms témoignent de variations, soulignant les enjeux et les rapports de force liés à ces reconfigurations (en termes de disciplines, d'ancrage territorial et de domaines de recherche).

Citons pour illustrer les principaux regroupements et leurs dénominations:

- exemple de fondation: «Paris Universitas »32 (janvier 2007) entre l'École des hautes études en sciences sociales (EHESS), l'École normale supérieure (ENS), les universités Paris II, III, VI et IX. «Paris Universitas » souhaite, avec ce nom, réincarner l'historique Université de Paris;

- exemples de PRES33 : en janvier 2006, PRES «Paris Centre Universités», regroupant les universités Paris I, V et VII34. En octobre 2009, PRES «La Sorbonne », regroupant les universités Paris II, IV et VI. En février 2010, PRES «Université Paris Cité », regroupant les universités Paris III, V, VII, l'École des hautes études en santé publique, l'Institut national des langues et civilisations orientales, l'Institut de physique du globe de Paris, l'Institut d'études politiques de Paris et l'université Paris XIII (membre associé), et dont le premier conseil d'administration (31 mars 2010) a symboliquement et stratégiquement adopté comme nom d'usage : PRES «Sorbonne Paris Cité»;

- Plan Campus, juillet 200835 : «Campus Condorcet Paris-Aubervilliers» (retenu en septembre 2009) entre l'École des hautes études en sciences sociales, l'École pratique des hautes études, les universités Paris I, VIII, XIII, l'École des Chartes, le CNRS et l'INED ; «Paris Campus Quartier Latin » (dossier déposé en juillet 2008) entre les universités Paris II, III, IV et VI, l'ENS Ulm et le Collège de France; «Campus universitaire Paris-Centre» (dossier déposé en juillet 2008) entre les universités Paris V et VII, l'IEP de Paris, l'Observatoire de Paris et l'Institut de physique du globe de Paris.

Une même université aux noms pluriels peut avoir rejoint plusieurs dispositifs (exemples: Paris I, II, III, V, VI...), ce qui conduit à une multiplication des signatures. Or, un des effets attendus de ces regroupements est de se voir attribuer plus de références, du fait de la logique bibliométrique, en fusionnant les signatures des contributions des chercheurs appartenant aux différents établissements et regroupés dans la nouvelle entité. Les signatures additionnées

32. «Fondation » est le terme utilisé pour désigner ce regroupement. Il s'agit cependant d'une association selon la loi de 1901.

33. Au total, les universités de Paris intra-muros disposent d'une dotation en capital de 700 millions d'euros dans le cadre de l'Opération Campus (dont 200 millions d'euros pour le PRES «Université Paris Cité »). Source : [www.gouvernement.fr], février 2010.

34. Par souci de lisibilité, nous avons indiqué la date de mise en place du PRES, le nom des établissements concernés en utilisant le chiffre romain dans le cas des universités et le nom administratif pour les instituts ou grandes écoles.

35. Les membres concernés peuvent varier selon les étapes de la procédure. Le cas de Paris intramuros est complexe et les regroupements n'ont pas encore été définis dans le cadre de l'opération Campus; voir site du ministère de l'Enseignement supérieur, dossier Opération campus [http://www.enseignementsup-recherche.gouv.fr/cid20924/operation-campus-renovationprojets-campus.html], consulté de novembre 2009 à mai 2010. 
par Paris I, V et VII dans le cadre du PRES «Paris Centre Universités » avaient pour but affiché d'augmenter la place de ces établissements dans les classements internationaux et les chiffres générés par les moteurs de recherche36. Une majorité d'établissements se préoccupent ainsi de leur notoriété et de leur «attractivité » au niveau national et international en veillant à l'affiliation et à la quantification correcte de leur signature. Le nom de l'établissement cristallise donc des enjeux en termes d'image mesurable et également de reconnaissance, dans une logique comptable de citation.

Rappelons que seules les références strictement identiques peuvent être comptabilisées - donc cumulées - et ainsi garantir une position enviable dans les classements internationaux37. Or, la succession de logiques de regroupement ne facilite pas la stabilisation des signatures et par voie de conséquence l'affiliation des références. Prenons l'exemple d'un établissement au nom pluriel : cette pluralité de dénominations pour une même entité conduit à un éclatement des références potentiellement attribuables, ce qui nuit à sa visibilité, les outils n'agrégeant pas systématiquement les différentes dénominations. L' «Université Paris Sud-11» est aussi appelée «Orsay», "ParisSud », "Sceaux»... De plus, la présence ou l'absence du trait d'union au sein des dénominations peut également être source d'éclatement d'affiliation des références.

Ainsi, dans le même but que celui des regroupements, les établissements qui ont changé de nom le justifient par la prise en compte d'enjeux de communication et d'internationalisation éducative. Par exemple, en novembre 2006 , Jean-François Dhainaut, le président de «Paris V - René Descartes», a été à l'initiative du nouveau nom, «Université Paris Descartes », et a justifié ce changement dans un courrier électronique du 31 juillet 2007 en rappelant «l'identité, la visibilité et l'attractivité nationale et internationale de [l']établissement. Notre université poursuit ainsi sa démarche fédérative autour d'un “label commun” à toutes les entités qui la composent» (nous soulignons).

Or, les changements de nom s'avèrent difficiles à imposer. Si les chercheurs sont sensibilisés à cette question, les contraintes de publication (feuille de style et charte graphique) et l'appartenance du chercheur à plusieurs structures peuvent participer à la non-affiliation de la référence. Par ailleurs, la prise en compte de l'enjeu d'affiliation par les chercheurs pose la question de leur

36. Sur les problèmes d'affiliation liés au classement de Shanghai, voir Liu, Cheng, 2005 et Zitt, Filliatreau, 2005.

37. En janvier 2010, le premier numéro de la lettre d'information du PRES «Université Paris-Est» indique sur sa page d'accueil : «En trois ans, neuf membres associés se sont rassemblés autour de nos cinq établissements fondateurs, regroupant ainsi tous les établissements publics concernés de l'Est francilien sous une même signature des publications scientifiques : “auteur, Université Paris-Est, laboratoire ou organisme, adresse” et pouvant utiliser une même adresse : prenom. nom@univ-paris-est.fr. » La place de cette information en page d'accueil confirme l'acuité du nom dans une logique de référencement. Source : [http://www.univ-paris-est.fr]. 
appartenance institutionnelle et du rapport du chercheur à ces identités imposées ou revendiquées (laboratoire et/ou UFR et/ou université et/ou organisme de recherche...).

Dans le cas de Paris V, l'élection d'un nouveau président en décembre 2007, Axel Kahn, a conduit à l'envoi par courrier électronique d'une note afin de demander aux chercheurs de l'établissement de signer leurs articles en reprenant le « label commun » prôné par son prédécesseur. Cette mobilisation quant à la signature institutionnelle des contributions scientifiques confirme la prise en compte d'enjeux de référencement - au cœur des questions liées à la compétitivité attendue du supérieur français - et leur difficile mise en pratique. Les classements internationaux sont explicitement évoqués dans cette note, ainsi que le «bon» référencement de l'établissement.

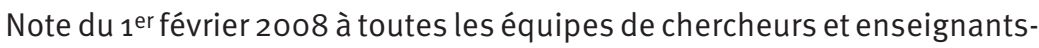
chercheurs :

Un usage croissant est fait des classements internationaux des établissements de recherche et d'enseignement supérieur. Le plus connu est celui de l'Université de Shanghai. Quels que soient l'analyse et le sentiment de chacun sur ces classements, ils jouent un rôle de plus en plus déterminant dans l'image des universités, et par conséquent leur attractivité, leur crédibilité, leur succès dans l'obtention de moyens auprès des instances nationales, européennes et internationales. [...] Des moteurs de recherche de différents organismes allouent automatiquement les publications aux établissements des auteurs À CONDITION QUE L'AFFILIATION SOIT CORRECTE. $» 38$

Une enquête effectuée dans le cadre d'un rapport sénatorial sur les classements internationaux dans le supérieur confirme l'importance accordée à ces classements. L'enquête, menée auprès de "106 dirigeants d'établissement d'enseignement supérieur »39, présente le taux global de réponse ( $75 \%)$ comme un signe d'intérêt porté aux classements 40 . Ainsi, $69 \%$ des répondants déclarent «connaître leur place dans le classement de Shanghai » $4^{11}$ et $83 \%$ des répondants déclarent également « avoir pris des mesures concrètes destinées à améliorer leur rang dans les classements internationaux».

Les changements de nom sont une «des mesures concrètes destinées à améliorer son rang ", comme l'attestent certains courriers ou notes internes. Si la stabilisation de la signature est la condition sine qua non du contrôle de la notoriété que nous qualifierons de «comptable», l'utilisation d'un nom prestigieux, devenu emblématique du champ académique, est également un moyen

38. Italiques : souligné par nous. Majuscules dans le texte.

39. " 84 universités et 22 grandes écoles», Rapport d'information sénatorial sur le "Défi des classements pour le supérieur», Joël Bourdin, juillet 2008, p. 97.

40. "79 réponses ont été reçues, soit un taux de réponse de $75 \%$, témoignant de l'intérêt des établissements interrogés pour les questions de l'évaluation et des classements. » Ibid., p. 97.

41. Ibid., p. 98. 
d'agir sur sa notoriété. Le cas de Paris est intéressant et mérite un examen attentif car parmi les treize universités étudiées, trois se partagent le nom Sorbonne (Paris I, III et IV) 42 et deux PRES l'emploient dans leur nom : PRES «La Sorbonne» et PRES «Sorbonne Paris Cité». L'intérêt pour l'usage du nom Sorbonne souligne le bénéfice attendu par son incontestable notoriété. Il indique également l’inscription de la dénomination académique dans le champ des marques.

\section{Signature, prestige et logique de marque. L'exemple de la Sorbonne}

La Sorbonne est l'emblème du rayonnement académique de la capitale et de la France. À l'instar de Louvre, Sorbonne est un toponyme43 qui est devenu au fil du temps le symbole de l'excellence académique française. Les ouvrages de marketing citent ainsi le Louvre et la Sorbonne comme des exemples de noms d'institutions devenues des marques (Heilbrunn, 2007, p.3) et répondant à deux fonctions essentielles : identification de l'origine (fonction de signature) et différenciation par rapport aux institutions concurrentes (ibid., p. 5.). L'un des emplois de «marque» est sémantiquement proche «d'un signe apposé intentionnellement», il renvoie également «aux armoiries, à l'ornement distinctif d'une dignité». Cet «ensemble de valeurs manifeste le lien entre la marque et la signature $» 44$.

Dans un contexte d'économie dite de la «connaissance», des signatures devenues des marques font l'objet de toutes les attentions de la part des pouvoirs publics. Ainsi, de nombreux groupes de travail ont été mis en place depuis 2007, principalement à l'initiative du ministère de l'Économie et des Finances, afin de recenser et de promouvoir des marques, appréhendées comme des actifs immatériels 45 . Cet intérêt porté aux marques s'observe dans le champ académique autour de la marque «Paris Sorbonne», déposée par la Ville de Paris en 2007. Or, l'histoire des universités parisiennes explique que les bâtiments de la Sorbonne abritent à la fois le rectorat de l'académie [http://www. sorbonne.fr] et des salles de cours ou de réunions de quatre établissements (Paris I, III, IV et V). Plusieurs universités se réclament donc de la Sorbonne. La constitution des PRES et le Plan Campus ont attisé les enjeux autour du nom Sorbonne et de l'utilisation de la marque.

Cette situation a conduit l'adjoint au maire de Paris, chargé de l’innovation,

42. "Université Paris 1 Panthéon-Sorbonne », «Université de Sorbonne Nouvelle Paris 3 » et «Université Paris Sorbonne - Paris IV ».

43. Construit à partir du patronyme de Robert de Sorbon (1201-1274), né à Sorbon (Ardennes), théologien, Maître à l'Université de Paris, fondateur du collège auquel il donna son nom.

44. Alain Rey, Dictionnaire historique de la langue française, vol. II, 2006, p. 2146.

45. Rapport Jouyet-Lévy sur l'économie de l'immatériel, décembre 2006. 
de la recherche et des universités $4^{6}$, à adresser une lettre, en juillet 2009, aux présidents des universités parisiennes afin de leur rappeler que «la Sorbonne est un des emblèmes de la vocation universitaire de la ville de Paris et un atout majeur pour l'ensemble des universités parisiennes. Le nom "Sorbonne" luimême est un élément important du patrimoine immatériel de la capitale, un bien commun de notoriété mondiale pour tous les Parisiens ». La marque et les bâtiments étant la propriété de la ville de Paris, l'adjoint au Maire indique : " Les événements récents, relatés par la presse, me conduisent à vous demander, au nom du Maire de Paris, d'utiliser le nom "Sorbonne" de manière concertée entre établissements et avec notre accord de propriétaire du bâtiment et de la marque.»

Ce rappel souligne les tensions suscitées par le feuilleté de signification lié au nom Sorbonne et dont de nombreux établissements souhaiteraient bénéficier. Les universités Paris I et Paris III ont conservé le nom Sorbonne dans leur nom officiel, mais il est accompagné pour Paris I de «Panthéon » et pour Paris III de «nouvelle». Seule Paris IV s'identifie exclusivement par le nom Sorbonne. Le choix de son URL en [www.paris-sorbonne.fr], proche de l'URL du rectorat, confirme l'enjeu accordé au nom Sorbonne et à la marque «Sorbonne».

Les sites web de plusieurs universités parisiennes, dans la partie consacrée à leur histoire, font ainsi référence à leur héritage vis-à-vis de la Sorbonne :

Héritière à la fois de la Sorbonne et de la Faculté de droit du Panthéon, Paris 1 est l'une des universités de premier plan dans le domaine des sciences humaines et sociales comme dans celui du droit et de l'économie. (Site de Paris l)

Héritière du Collège fondé en 1257 par Robert de Sorbon à l'emplacement de l'actuelle Sorbonne, puis de la Faculté des Lettres de l'Université de Paris, l'Université Paris-Sorbonne (Paris IV) a été créée par le décret du 23 décembre 1970, confirmé par le décret du 17 juillet 1984. (Site de Paris IV)

La logique de marque, voire de label a ainsi conduit l'«Université ParisSorbonne, Paris IV» à ouvrir un campus à Abu Dhabi, « Paris-Sorbonne University Abu Dhabi », qui affiche le même logo que celui qui figure sur le site parisien (représentation de la chapelle de la Sorbonne). La mention «Abu Dhabi » figurant en bas du logo permet de distinguer les deux entités de la marque "Sorbonne». Inauguré le 18 novembre 2006 par Gilles de Robien, alors ministre de l'Éducation nationale, ce campus est censé incarner le dynamisme des marques françaises à l'exportation.

La résonance dans les champs politique, médiatique et académique de l'importance accordée aux classements internationaux, en particulier au classement de Shanghai, inscrit donc la question de la visibilité et de la notoriété des dénominations académiques au cœur des stratégies d'établissements dans le but affiché d'agir sur les logiques comptables d'affiliation. Cependant,

46. Courrier de Jean-Louis Missika du $1^{\mathrm{er}}$ juillet 2009. 
indépendamment de la compétitivité attendue, des stratégies de communication et de la logique de marque, des pratiques discursives de signatures plurielles demeurent et expliquent la coprésence de plusieurs noms pour une même université, voire un PRES. La multiplication des dispositifs de regroupement ne favorise pas la stabilisation d'un nom, ce qui conduit à un certain brouillage quant à la visibilité attendue des universités françaises. La mobilisation reste cependant forte pour améliorer le classement des universités parisiennes et favoriser leur «notoriété », confirmant la diffusion d'un discours de l'excellence qui tend à s'imposer et à résumer l'excellence à des logiques quantitatives d'affiliation ou de marque.

\section{Références}

Amossy Ruth éd., 1999, Images de soi dans le discours. La construction de l'ethos, Lausanne, Delachaux et Niestlé.

Austin John Langshaw, 1962, How to do Things with Words, Oxford, Clarendon Press (Quand dire c'est faire, Paris, Seuil, 1970).

BACOT Paul, Douzou Laurent, HonorÉ Jean-Paul éd., 2008, Mots. Les langages du politique, n०87, Chrononymes. La politisation du temps.

BARATS Christine, 2008, «Les mots de l'internationalisation éducative. Rhétorique ministérielle sur la "compétitivité” et l'“attractivité" des universités parisiennes", Jocair'08, Paris, Hermès Lavoisier, p. 365-379.

- à paraître, «Rhétorique de l'accueil académique. Pour une lecture comparée des sites universitaires de l'île-de-France », Congrès SFSIC, Dijon, 23-25 juin 2010.

BARTHes Roland, 1967, «Proust et les noms », Le degrézéro de l'écriture, Paris, Seuil, 1972.

Boyer Henri, Paveau Marie-Anne éd., 2008, Mots. Les langages du politique, $\mathrm{n}^{\circ} 86$, Toponymes. Instruments et enjeux.

BRUNo Isabelle, 2008, À vos marques, prêts cherchez! La stratégie européenne de Lisbonne, vers un marché de la recherche, Paris, Croquant (Savoir/Agir).

Fou CaUlt Michel, 1971, L'ordre du discours, Paris, Gallimard.

GINGRAT Yves, 2008, La fièvre de l'évaluation de la recherche. Du mauvais usage de faux indicateurs, Note de recherche, Québec, Montréal, CIRST, UQAM.

Goody Jack, 1979, La raison graphique, Paris, Minuit.

Honoré Jean-Paul, Paveau Marie-Anne, Périès Gabriel éd., 2000, Mots. Les langages du politique, $\mathrm{n}^{\circ} 63$, Noms propres.

HeIlbrunn Benoît, 2007, La marque, Paris, PUF (Que sais-je?).

LIU Nian Cai, CHENG Ying, 2005, "Academic ranking of world universities. Methodologies and problems », Higher Education in Europe, vol.XXX, $\mathrm{n}^{\circ} 2$, Unesco-CEPES, en ligne [http://www.cepes.ro/publications/pdf/hee_eng_pdf/he2_05.pdf]. Consulté le 7 octobre 2010.

MIEG Bernard, 2007, La société conquise par la communication, t. III, Les Tic entre innovation technique et ancrage social, Grenoble, Presses universitaires de Grenoble. 
MUSSELIN Christine, 2001, La longue marche des universités françaises, Paris, PUF.

Paveau Marie-Anne, Reboul-Touré Sandrine, Lecolle Michelle éd., 2009, Carnets du Cédiscor, $\mathrm{n}^{\circ}{ }^{11}$, Le nom propre en discours.

Zitt Michel, Filliatreau Ghislaine, 2005, «Big is (made) beautiful. Some comments about the Shanghai ranking of world-class universities», en ligne [http://www. cepes.ro/publications/WCU/contents.html]. Consulté le 8 octobre 2010. 


\section{Annexe}

\begin{tabular}{|c|c|c|}
\hline $\begin{array}{l}\text { Noms employés } \\
\text { dans cet article }\end{array}$ & $\begin{array}{l}\text { Noms à la date de création } \\
\text { (corpus : arrêtés d'approbation } \\
\text { des statuts, 1970) }\end{array}$ & $\begin{array}{l}\text { Noms et caractéristiques } \\
\text { morphosyntaxiques } \\
\text { (corpus : sites web, avril 2010) }\end{array}$ \\
\hline Paris I & $\begin{array}{l}\text { Université de Paris-I } \\
\text { (Panthéon-Sorbonne- } \\
\text { Sciences économiques-Sciences } \\
\text { humaines-Sciences juridiques) } \\
\text { Arrêté du } 14 \text { décembre } 1970\end{array}$ & $\begin{array}{l}\text { Université Paris 1, Panthéon Sorbonne } \\
\text { Chiffre arabe }+ \text { lieu }\end{array}$ \\
\hline Paris II & $\begin{array}{l}\text { Université de droit, d'économie } \\
\text { et de sciences sociales } \\
\text { (Paris-II) et Université de Paris-II } \\
\text { Arrêté du } 19 \text { novembre } 1970\end{array}$ & $\begin{array}{l}\text { Université Panthéon-Assas } \\
\text { Lieu }\end{array}$ \\
\hline Paris III & $\begin{array}{l}\text { Université de Paris-III } \\
\text { Arrêté du } 26 \text { novembre } 1970\end{array}$ & $\begin{array}{l}\text { Université Sorbonne Nouvelle Paris } 3 \\
\text { Lieu + chiffre arabe }\end{array}$ \\
\hline Paris IV & $\begin{array}{l}\text { Université de Paris-Sorbonne (Paris-IV) } \\
\text { Arrêté du } 8 \text { décembre } 1970\end{array}$ & $\begin{array}{l}\text { Université Paris Sorbonne-Paris IV } \\
\text { Lieu + chiffre romain }\end{array}$ \\
\hline Paris V & $\begin{array}{l}\text { Université René-Descartes de Paris } \\
\text { (Paris-V) } \\
\text { Titre : Approbation des statuts } \\
\text { de l'Université de Paris (Paris-V) } \\
\text { Arrêté du } 28 \text { octobre } 1970\end{array}$ & $\begin{array}{l}\text { Université Paris Descartes } \\
\text { Philosophe }\end{array}$ \\
\hline Paris VI & $\begin{array}{l}\text { Université de Paris-VI } \\
\text { Décret du } 23 \text { décembre } 1970 \\
\text { dotant de statuts }\end{array}$ & $\begin{array}{l}\text { Université Pierre et Marie Curie, UPMC } \\
\text { Scientifiques + sigle }\end{array}$ \\
\hline Paris VII & $\begin{array}{l}\text { Université de Paris-VII } \\
\text { Arrêté du } 14 \text { décembre } 1970\end{array}$ & $\begin{array}{l}\text { Université Paris Diderot, Paris } 7 \\
\text { Philosophe + chiffre arabe }\end{array}$ \\
\hline Paris VIII & $\begin{array}{l}\text { Université de Paris-Vincennes } \\
\text { (Paris-VIII) } \\
\text { Arrêté du } 19 \text { novembre } 1970\end{array}$ & $\begin{array}{l}\text { Université Paris } 8 \text {, Vincennes, } \\
\text { Saint Denis } \\
\text { Chiffre arabe + lieu }\end{array}$ \\
\hline Paris IX & $\begin{array}{l}\text { Université de Paris-Dauphine (Paris-IX) } \\
\text { Arrêté du } 30 \text { novembre } 1970\end{array}$ & $\begin{array}{l}\text { Université Paris Dauphine } \\
\text { Lieu }\end{array}$ \\
\hline Paris X & $\begin{array}{l}\text { Université de Paris-Nanterre (Paris-X) } \\
\text { Arrêté du } 24 \text { décembre } 1970\end{array}$ & $\begin{array}{l}\text { Université Paris Ouest Nanterre la } \\
\text { Défense } \\
\text { Lieu }\end{array}$ \\
\hline Paris XI & $\begin{array}{l}\text { Université de Paris-Sud (Paris-XI) } \\
\text { Arrêté du } 6 \text { novembre } 1970\end{array}$ & $\begin{array}{l}\text { Université Paris-Sud } 11 \\
\text { Lieu + chiffre arabe }\end{array}$ \\
\hline Paris XII & $\begin{array}{l}\text { Université de Paris Val-de-Marne } \\
\text { (Paris XII) } \\
\text { Arrêté du } 13 \text { novembre } 1970\end{array}$ & $\begin{array}{l}\text { Université Paris-Est Créteil Val } \\
\text { de Marne, UPEC } \\
\text { Lieu + sigle }\end{array}$ \\
\hline Paris XIII & $\begin{array}{l}\text { Université de Paris-Nord (Paris XIII) } \\
\text { Arrêté du } 13 \text { novembre } 1970\end{array}$ & $\begin{array}{l}\text { Université Paris-Nord } 13, \text { UP13 } \\
\text { Lieu }+ \text { chiffre arabe + sigle }\end{array}$ \\
\hline
\end{tabular}

Synthèse des principaux noms des 13 universités parisiennes (Arrêté d'approbation des statuts et supports de communication, 1970-2010) 